\title{
Do conflict-affected societies need psychiatrists? ${ }^{\dagger}$
}

\author{
Derrick Silove
}

\section{Summary}

Symptoms of post-traumatic stress disorder (PTSD), anxiety and depression are prevalent among post-conflict populations worldwide but controversy about the cultural meaning of these symptoms, particularly in relation to PTSD, adds to the challenge for psychiatry in mounting appropriate intervention programmes. Accumulating scientific evidence assists in guiding the focus and scope of emergency mental health programmes, initiatives that can become the vanguard of mental health development and reform for countries recovering from conflict.

\section{Declaration of interest}

None.
Derrick Silove has, for the past 25 years, worked with refugee and postconflict populations and other groups affected by trauma and human rights violations.

The paper by Kohrt et al ${ }^{1}$ exemplifies the advances that have been made in epidemiological research in the field of post-conflict mental health. Although a large body of research has revealed consistent associations between war-related trauma and mental disorders such as post-traumatic stress disorder (PTSD) and depression, ${ }^{2}$ the majority of studies have been cross-sectional in design, thereby limiting the causal inferences that can be drawn. The novelty of the study of Kohrt et $a l^{1}$ is that it recorded data before and after a period of conflict; of interest is that anxiety increased after war exposure but high levels of depression remained constant, being closely related to persisting conditions of poverty. The study provides a clear reminder of the inextricable link between the global challenges of mass conflict and poverty and common mental disorders that make a major contribution to the global burden of disease.

\section{Consensus and challenges}

The high prevalence of mental disorder in conflict-affected countries poses a major dilemma for psychiatry in devising appropriate responses, especially given that most of these societies have very low resources in mental health. Adding to the challenge is the ongoing controversy about the status of PTSD and the legitimacy of applying Western-derived trauma-focused therapies across diverse cultural settings, a debate that continues to divide leaders in the field. ${ }^{3}$ Yet organisations such as the World Health Organization (WHO), the United Nations High Commissioner for Refugees and the World Psychiatric Association, working with non-governmental organisations and other partners, have managed to forge a consensus about the core principles of policy and practice that should be applied in the post-conflict mental health field. ${ }^{4-6}$ Perhaps sensibly, this consensus has been reached largely by ignoring the thorny issue of PTSD.

\section{Scientific evidence concerning trauma and PTSD}

There now is a large body of epidemiological research that allows us to begin answering some of the controversial issues about

†See pp. 268-275, this issue
PTSD in conflict-affected countries. Studies show that PTSD can be detected across a wide range of cultural settings even though the prevalence of the disorder varies substantially across countries. ${ }^{2}$ Some of this variation is attributable to the research methodologies employed; larger, random samples using structured clinical interviews yield much lower rates of PTSD than smaller, non-random studies applying self-report questionnaires. ${ }^{2}$ There also is overwhelming evidence that the quantum and type of trauma exposure are key factors in determining prevalence rates of PTSD across studies. ${ }^{2}$ In particular, the impact on mental health of exposure to human rights trauma such as torture, rape and other forms of interpersonal abuse is more severe and long-lasting than that of accidents and natural disasters.

Culture is commonly invoked to explain differences in PTSD rates across countries but it may be that the particularities of the historical context and the social impact of conflict are at least as relevant. For example, studies among Cambodians have yielded consistently higher rates of PTSD compared with the Vietnamese, even though the wars in these neighbouring countries occurred during the same time epochs. ${ }^{2}$ In Cambodia, the Khmer Rouge transformed the whole country into a virtual concentration camp, destroying existing institutions and social networks and making it all but impossible to avoid exposure to multiple threats and abuses, whereas in Vietnam, the war had a patchy geographical impact so that substantial sectors of the population were spared direct exposure to violence and associated disruptions of social structures and networks. ${ }^{7}$

The timing of studies following the peak period of conflict has emerged as an important predictor of PTSD prevalence rates across countries. ${ }^{2}$ Stress symptoms tend to be high in the midst of conflict and in the immediate aftermath - in that sense, symptom levels provide an accurate barometer of the level of communal terror and arousal in the acute phase. Fortunately, time is a good healer for the population as a whole, ${ }^{2}$ as long as the recovery environment is stable and supportive - sadly, conditions that rarely pertain in the immediate post-conflict phase in the contemporary world.

\section{From science to policy}

These and other scientific observations help to shape the core principles that guide contemporary mental health programmes in low-income, post-conflict countries. There is a consensus across the field of psychiatric traumatology that early debriefing of entire populations exposed to disasters is ineffective and may be 
damaging to some survivors. ${ }^{8}$ In the immediate post-conflict phase, the best treatment is social - that is, creating conditions of safety, stability and socioeconomic development that will hasten natural recovery for the majority of the population. An important qualification, however, is that there will be a minority of survivors who have experienced overwhelming levels of violence and abuse and who will continue to experience a chronic form of PTSD, commonly comorbid with other disorders such as depression. These problems often become evident at the very point that international aid programmes begin to wind down.

Importantly, there is accumulating evidence that culturally adapted trauma-focused cognitive-behavioural therapy can be applied effectively across cultures, even though these interventions are seldom used. ${ }^{6}$ Further trials are needed to examine why the uptake of these treatments is so low, what the effective components of these therapies are, who will benefit most from these interventions, the contraindications to exposing some survivors to memories of abuse such as torture, and the psychological impact on non-professional therapists (often survivors themselves) of listening repeatedly to harrowing accounts of violence. Standards need to be established for training therapists, assessing their competency and providing adequate ongoing supervision.

\section{Range of disorders and cultural expressions of distress}

Although PTSD remains central to work in post-conflict settings, the overweening preoccupation with that single diagnostic category has had the undesirable effect of diverting attention from a range of other psychiatric disorders including depression, various subtypes of anxiety (including separation anxiety), explosive forms of anger and other impulse control problems, prolonged grief, somatoform complaints, substance and alcohol misuse, personality change and head injury. It is remarkable too how little research has been conducted among persons with psychotic illnesses in the post-conflict field, a gap that has only begun to be addressed recently. For example, a study in postconflict Timor Leste found that psychotic disorders are at least as common and disabling in that setting as in high-income countries. $^{9}$

Epidemiological studies also have been remiss in ignoring cultural expressions of distress among conflict-affected populations. For example, a study among a large sample of waraffected Vietnamese found that an indigenously derived measure of anxiety, depression and somatic symptoms identified three times the number of cases of mental disorder as the widely used Composite International Diagnostic Instrument. ${ }^{7}$ The lesson seems to be clear: a sole reliance on international measures of psychiatric disorder may lead to an underestimate of the need for services in post-conflict societies. A key challenge for future epidemiological research is to explore further the bridging points between the universal (etic) and the local (emic) representations of emotional responses to trauma and abuse, an enterprise that requires a painstaking methodological approach. ${ }^{10}$

\section{Principles guiding emergency programmes}

How do these considerations guide us in designing mental health programmes for low-income, conflict-affected countries, particularly in the early post-conflict phase? Given the multiplicity of psychiatric problems that can be triggered by trauma and conflict, it does not make sense to prioritise a single diagnosis such as PTSD. The key criterion for priority attention should be the level of social vulnerability - that is, the extent to which the presence of mental disorder is undermining the capacity of the individual, and indirectly the family, to survive and adapt in the precarious conditions that prevail in post-conflict environments. ${ }^{11}$ From a practical perspective, this means that priority should be given to those with mental disorder who are suicidal or a threat to others, who are disruptive to families and communities, and/or who are so disabled that they cannot care for themselves or their dependents. ${ }^{11}$

There now is a legacy of work in establishing family-focused, community-based mental health services in post-conflict settings, low-cost programmes that require little in the way of technology or infrastructure to initiate. ${ }^{11} \mathrm{~A}$ forthcoming publication by WHO will provide a series of case examples that will illustrate the application of these models across diverse post-conflict settings around the world. ${ }^{12}$ These vanguard programmes can act as the catalyst for the reform of mental health services as conflict-affected countries enter the phase of reconstruction. ${ }^{4}$ By highlighting the effectiveness of a community-focused approach, emergency programmes can help steer policies and practices away from previously entrenched custodial care models often inherited from the colonial period. In that way, the convulsion of conflict, although always destructive and never desirable, can bring in its wake an impetus towards mental health system reform and development, a transformation that can have a lasting impact on the society for years to come. ${ }^{4}$

Controversy is inevitable in a field that spans the domains of human rights, international politics, conflict theory, transcultural psychiatry and psychiatric traumatology. There may be some legitimacy in the concern that culturally naive psychiatrists can become the unwitting stalking horses of neocolonial designs, colonising minds rather than territory. At the same time, those who argue for a 'hands-off' approach to psychiatric intervention in conflict-affected societies are at risk of over-arguing their case. The stark reality is that there are significant numbers of conflict survivors worldwide who receive no effective support or treatment for their severe mental disorders. In my experience, these people and their families are desperately concerned with obtaining relief from their suffering - and much less interested in the ideological debates that are current in the Western-dominated literature.

Derrick Silove, MD, FRANZCP, Psychiatry Research and Teaching Unit, Level 1 Mental Health Centre, Liverpool Hospital, Liverpool, NSW 2170, Australia. Email: d.silove@unsw.edu.au

First received 20 Mar 2012, final revision 19 Jul 2012, accepted 13 Aug 2012

\section{References}

1 Kohrt BA, Hruschka DJ, Worthman CM, Kunz RD, Baldwin JL, Upadhaya N, et al. Political violence and mental health in Nepal: prospective study. $\mathrm{Br} J$ Psychiatry 2012; 201: 268-75.

2 Steel Z, Chey $T$, Silove D, Marnane C, Bryant RA, van Ommeren M. Association of torture and other potentially traumatic events with mental health outcomes among populations exposed to mass conflict and displacement: a systematic review and meta-analysis. JAMA 2009; 302: $537-49$

3 Summerfield D. How scientifically valid is the knowledge base of global mental health? BMJ 2008; 336: 992-4.

4 Inter-Agency Standing Committee. IASC Guidelines on Mental Health and Psychosocial Support in Emergency Settings. Inter-Agency Standing Committee, 2007.

5 Mezzich J, Saraceno B. The WPA-WHO Joint Statement on the Role of Psychiatrists in Disasters Response. World Psychiatry 2007; 6: 1-2.

6 Tol WA, Barbui C, Galappatti A, Silove D, Betancourt TS, Souza R, et al. Mental health and psychosocial support in humanitarian settings: Linking practice and research. Lancet 2011; 378: 1581-91. 
7 Steel Z, Silove D, Giao NM, Phan TTB, Chey T, Whelan A, et al. International and indigenous diagnoses of mental disorder among Vietnamese living in Vietnam and Australia. Br J Psychiatry 2009; 194: 326-33.

8 McNally $R$, Bryant $R$, Ehlers A. Does early psychological intervention promote recovery from posttraumatic stress? Psychol Sci Public Interest 2003; 4: 45-79.

9 Silove $\mathrm{D}$, Bateman $\mathrm{CR}$, Brooks RT, Fonseca AZ, Steel Z, Rodger J, et al. Estimating clinically relevant mental disorders in a rural and an urban setting in postconflict Timor Leste. Arch Gen Psychiatry 2008; 65: 1205-12.
10 van Ommeren $\mathrm{M}$. Validity issues in transcultural epidemiology. $\mathrm{Br} \mathrm{J}$ Psychiatry 2003; 182: 376-8.

11 Silove D, Manicavasagar V, Baker K, Mausiri M, De Carvalho F, Soares A, et al. Indices of social risk among first attenders of an emergency mental health service in post-conflict East Timor: an exploratory investigation. Aust N Z Psychiatry 2004; 38: 929-32.

12 World Health Organization. Building Back Better: Sustainable Mental Health Care After Disaster. WHO, in press.

\section{Reflections}

\section{Margaret Theresa Carney}

I think that at the beginning,

of madness I was very small.

Small and lost in a paranoid world.

The voices taunted me and people mocked.

Oh I saw them all, all the people in the white suits.

I ranged the highway lost inside myself.

Reality did not seem real, it was too hard.

I spent Christmas in an institution.

We drank tea and we were casualties

Accidents of life, a death, an illness

Loneliness - the Lavender lace of solitude.

I tried to reach out but there was a screen,

A screen of broken images

Silhouettes and Flashes, illusion, illusion,

Memories and fantasies all overgrown.

Dad says I get by,

They have stopped putting me away,

I moved and I got a little house.

And I fought like a tigress

To keep it together.

The mind can be a terrible thing

Untethered, let free.

But at last I did love myself,

I did finally love myself,

And I stood alone, on a great dark cliff

And I called the wild dark seas

I called them to my breast.

I am a poet

And the words fell like blood drops

From a large soul.

God loves me now.

This poem is from Margaret Theresa Carney's book Tales from the Womb, published in 2006 by Survivors' Poetry. Carney was mentored by Paula Brown.

Chosen by Femi Oyebode. 